\title{
Non-local high cycle fatigue criterion for metallic materials with corrosion defects
}

\author{
Mohamed El May ${ }^{1, \mathrm{a}}$, Nicolas Saintier ${ }^{1}$, Thierry Palin-Luc ${ }^{1}$ and Olivier Devos ${ }^{2}$ \\ ${ }^{1}$ Arts et Métiers ParisTech, I2M, CNRS UMR 5295, 33405 Talence Cedex, France \\ ${ }^{2}$ Université de Bordeaux, I2M, CNRS UMR 5295, 33405 Talence Cedex, France
}

\begin{abstract}
Designing structures against corrosion fatigue has become a key problem for many engineering structures evolving in complex environmental conditions of humidity (aeronautics, civil engineering ...). In this study, we investigate the effect of corrosion defects on the high cycle fatigue (HCF) strength of a martensitic stainless steel with high specific mechanical strength, used in aeronautic applications. A volumetric approach based on Crossland equivalent stress is proposed. This can be applied to any real defects.
\end{abstract}

\section{Introduction}

Several fatigue strength criteria have been developed to account for the effect of a geometrical defect on the fatigue strength [1-3]. These methods are often applied in the case of idealized geometries of defects (hemispherical, ellipsoids ... etc.). They are mainly not applicable to the irregular geometries of real defects or on complex component geometry since stress gradient approaches require a 2D approximation of the defects geometry [2]. The purpose of this study is to propose a non-local fatigue strength criterion able account for real geometries and distributions of corrosion defects.

\section{Experimental results}

The fatigue strength at $10^{7}$ cycles under fully reserved tension was determined in air on virgin and pre-corroded specimens made of $\mathrm{X} 13 \mathrm{CrNiMoV} 12-3$ stainless steel. Pitting was performed at imposed electrochemical potential, so that four controlled sizes defects were obtained. Consequently an experimental Kitagawa diagram [4], representative of real corrosion pits (not micro-machining) was obtained. A minimum of five specimens by defect size were tested to identify the experimental fatigue limit at $10^{7}$ cycles of the X13CrNiMoV12-3 stainless steel, using the short stair case method [5]. The empirical $\sqrt{ }$ area fatigue criterion of Murakami [3] provides a safe estimation of the experimental fatigue limit but is difficult to apply when the local stress state is multiaxial.

\footnotetext{
${ }^{a}$ Corresponding author: mohamed.elmay@ensam.eu

This is an Open Access article distributed under the terms of the Creative Commons Attribution License 4.0, which permits unrestricted use, distribution, and reproduction in any medium, provided the original work is properly cited.
} 


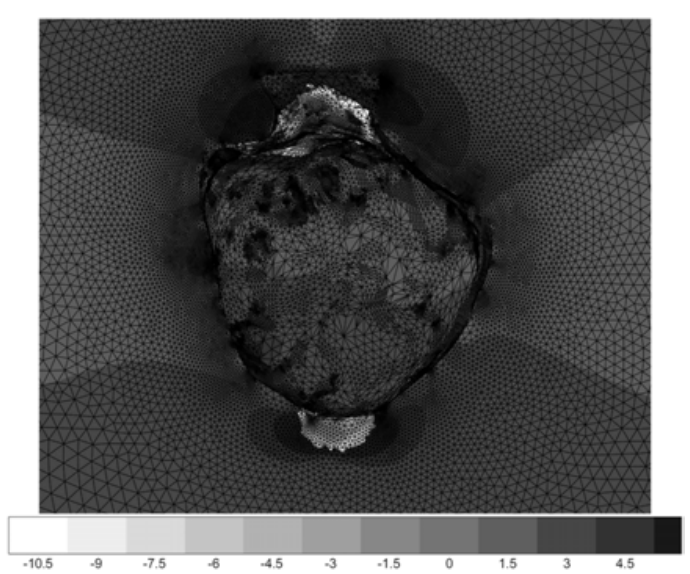

Figure 1. $\left(\sigma_{\text {mises }} / \sigma_{H}\right)$ local distribution of a real corrosion pit identified by X-ray microtomography.

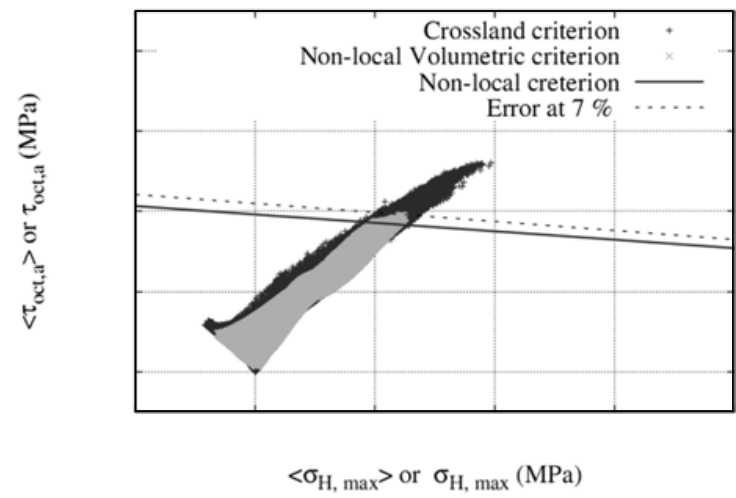

Figure 2. FE simulation of natural pit $(\sqrt{\text { area }}=266 \mu \mathrm{m})$ with $\sigma_{n o m, a}=\sigma_{(R=-1)}^{D}$.

\section{Non-local fatigue criterion}

Elastic-plastic numerical simulations were performed for real defect geometry, identified by X-ray microtomography, to determine the local stress distribution. The analysis revealed that calculation of the fatigue strength for material with real (irregular) pit geometry required a non-local HCF strength criterion. The local distribution of $\left(\sigma_{\text {mises }} / \sigma_{H}\right)$ revealed a local multiaxial stress (Fig. 1).

To account for defects the proposed criterion is based on the Crossland equivalent stress averaged in the zone affected by the defect $\left(Z_{d e f}\right)$, limited by $\sqrt{\text { area }}$ [2], within a volume $(V)$ limited by a critical distance $\left(d_{c}\right)[5]$.

$$
\begin{gathered}
\max _{M \in Z_{d e f}}\left\lfloor<\tau_{o c t, a}>+\alpha<\sigma_{H, \max }>\right\rfloor \leq \beta \\
<\tau_{o c t, a}>=\frac{1}{v} \iiint_{v} \tau_{o c t, a} d V \\
<\sigma_{H, \max }>=\frac{1}{v} \iiint_{v} \sigma_{H, \max } d V .
\end{gathered}
$$

The parameters $(\alpha)$ and $(\beta)$ of the Crossland criterion were identified by fatigue tests in air, on the base material without corrosion defect, for two loading ratios $(R=-1$ and $R=0.1)$. The critical distance $\left(d_{c}\right)$ was optimized at $7 \mu \mathrm{m}$ using fatigue results of only one size of defects $(\sqrt{\text { area }}=33 \mu \mathrm{m})$. Currently, no obvious relationship was found between the value of $\left(d_{c}\right)$ and the microstructure of the material. This criterion was validated with HCF tests on specimens with natural corrosion defects corresponding to a larger defect size $(\sqrt{\text { area }}=266 \mu \mathrm{m})$ also identified from X-ray microtomography (Fig. 2).

\section{Conclusion}

This criterion was validated with natural corrosion defects on $\mathrm{X} 13 \mathrm{CrNiMoV} 12-3$ martensitic stainless steel tested under uniaxial fatigue loading. Other material testing and multiaxial fatigue loading should be studied to confirm the prediction capabilities of the criterion. 


\section{References}

[1] N. Saintier, T. Palin-Luc, J. Bénabes et F. Cocheteux, Int. J. of Fatigue (2013)

[2] H. Gadouini, Y. Nadot and C. Rebours, Int. J. of Fatigue (2008)

[3] Y. Murakami, Metal fatigue: Effects of small defects...(Elsevier ed., 2002)

[4] H. Kitagawa and S. Takahashi, $2^{\text {nd }}$ In. conf. mech. behavior of materials (1976)

[5] M. El May, Phd Thesis, Arts et Métiers ParisTech (2013) 